\title{
Development of an Enzyme-Linked Immunosorbent Assay for quantification of Salmonella Enteritidis-specific antibodies in egg yolk
}

- Author(s)
Tayeb $\mathrm{IT}^{1}$
Nehme $\mathrm{P}^{2}$
Jaber $\mathrm{L}^{2}$
Barbour EK ${ }^{2 *}$
Department of Animal Production, College
of Agriculture, University of Dhouk,
Kurdistan region, Iraq.
2 Department of Animal Sciences, Faculty of
Agricultural and Food Sciences, American
University of Beirut, Beirut, Lebanon.

Mail Address

EK Barbour

Department of Animal Sciences

Faculty of Agricultural and Food Sciences,

American University of Beirut

Beirut, Lebanon

Tel: 961-1-350000, ext: 4460

Fax: $961-1-744460$

E-mail: eb01@aub.edu.lb

\section{Keywords}

Antibodies; egg yolk; ELISA; Salmonella Enteritidis.

\section{Abbreviations}

BSA, bovine serum albumin; CFU, colony forming units; ELISA, enzyme-linked immunosorbent assay; PBS, phosphate buffered saline; SE, Salmonella Enteritidis.

\section{ABSTRACT}

The present study aims at developing an indirect ELISA to quantify yolk antibodies specific to all surface proteins of the invasive Salmonella Enteritidis (SE), which acquired the 1.8, 14.1, and $50 \mathrm{~Kb}$ plasmids. An ELISA checkerboard was used in four different experiments to account for the different parameters included in the preliminary ELISA procedure, and consequently to maximize the difference in Optical Density (OD) values between control positive and negative yolk samples. The first experiment aimed at studying the impact of 5\% Bovine Serum Albumin (BSA) dissolved in distilled water as a blocking reagent on a $28 \mu \mathrm{g} / \mathrm{well}$ SE antigen-coated plate, while applying the positive and negative control yolk samples to different concentrations of Phosphate-Buffered Saline (PBS). Conjugate application was maintained constant at a dilution of 1:500 in PBS. The second experiment was similar to the first one, but the positive and negative control yolk samples were diluted in PBSTween 20, and the conjugate dilution was changed to 1:1500 in PBSTween 20. In the third experiment, the conjugate was diluted at 1:1500 in 5\% BSA/PBS-Tween 20 diluent or PBS-Tween 20 diluent with no 5\% BSA. The objective of the fourth experiment was to study the impact of four different concentrations of SE-coated antigen levels $(28 \mu \mathrm{g} /$ well, $56 \mu \mathrm{g} / \mathrm{well}, 84 \mu \mathrm{g} / \mathrm{well}$, and $112 \mu \mathrm{g} / \mathrm{well})$, while fixing the blocking step with 5\% BSA in distilled water, and the conjugate dilution set at 1:1000 in 5\% BSA/PBS-Tween 20, and fixing the control yolk samples dilution at $1 \%$ in PBS-Tween 20. This last experimental procedure allowed the highest difference in mean absorbance OD values of the positive control minus the negative control samples, which was equivalent to 0.381 . In addition, the final protocol for this ELISA was applied on individual egg yolk samples of two groups of chicken layers: one challenged in the esophagus at 11 days with $5.4 \times 10^{10} \mathrm{CFU} / \mathrm{ml} / \mathrm{bird}$ of SE, and the second group was not challenged. The mean OD values of the egg yolk of antibodies specific against SE of the two groups were significantly different $(0.8578$ versus $0.5250 ; p<0.05)$, which indicates the possible application of the developed ELISA for screening SE infection by examining egg yolks produced by commercial layers.

\section{INTRODUCTION}

Salmonella Enteritidis (SE) acquiring a high molecular weight plasmid $(\sim 50 \mathrm{~Kb})$ is reported as a highly invasive pathogen, resulting in significant damage in avian and human hosts (Barbour et al., 1999; Saeed and Nair, 1999). SE was found to be the most common Salmonella serotype recovered from human cases in the USA, accounting for $76 \%$ of all septicemia cases and 39\% of all cases of gastroenteritis (Gruenewald et al., 1999). In poultry, the invasive SE causes more damage in young chicks as compared to adults (Gast and Beard, 1989; Uyttebroek et al., 
1991). Natural infection by invasive SE causes the following lesions in young chicks: namely, fibrinous pericarditis, airsacculitis, perihepatitis, peritonitis, cecal cores, and unabsorbed yolk sacs (Gorham et al., 1994). The pathogenesis of invasive SE in adult chicken layers is milder, resulting in slight heterophil infiltration in the ovaries and oviducts (Hoop \& Pospischil, 1993).

The classical approach followed in many investigations of SE outbreaks in poultry relies mainly on culturing the egg contents for SE isolation (yolk, yolk vitelline membrane, albumen). This approach is time-consuming, and can be misleading due to the very low frequency of SE- infected eggs (Dadrast et al., 1990). This fact led many researchers around the world to develop indirect ELISA for the identification of SEspecific antibodies in the egg yolk in order to assess the infectivity status of poultry flocks (Nicholas \& Cullen, 1991; Furrer et al., 1993; Barrow, 1994; Desmidt et al., 1996; Dera-Tomaszewska et al., 2003). In addition, scientists are targeting the development of the indirect ELISA for the analysis of SE-specific antibodies in the egg yolk instead of serum SE antibodies, thus avoiding the stress induced by handling birds during blood collection, and at the same time, abiding to the biosafety protocols of the farms included in national disease control programs. The nature of the coating of the ELISA plates for SE-specific antibody quantification varies among the scientists due to the difference in the prevalent strain(s) of SE, or due to interests of the scientists in quantification of certain protective antibodies against specific antigens in the SE, such as fimbriae or pilli, flagella, lipopolysaccharide, heat shock protein (HSP 60), or all surface proteins of SE (Dadrast et al., 1990; Nicholas \& Cuellen, 1991; Dera-Tomaszewska et al., 2003; Oliveira et al., 2004). The prevalent invasive SE in the poultry of Lebanon is the one that acquired three plasmids, namely, 1.8, 14.1, and $\sim 50 \mathrm{~Kb}$ (Barbour et al., 1999). Other reports from around the world report the presence of the high molecular weight plasmid of $\sim 50 \mathrm{~Kb}$, which is responsible for the invasiveness of particular SE strains (Guard-Petter, 1998; Guard-Petter et al., 1999; Van Asten and Van Dijk, 2005).

The purpose of this work was to develop an indirect ELISA to quantify yolk antibodies specific to all surface proteins of the invasive SE that acquired the 1.8, 14.1 and $\sim 50 \mathrm{~Kb}$.

\section{MATERIALS AND METHODS}

\section{Reagents in preliminary ELISA procedure}

Salmonella Enteritidis cells were harvested from Nutrient Agar plates, solubilized, and charged by the addition of Sodium Dodecyl Sulphate (SDS) in a w/w ratio of SDS/Salmonella Enteritidis proteins equivalent to $1: 29.1$. The charged antigen stock was incubated at $37^{\circ} \mathrm{C}$ for 2 hours under continuous stirring. The antigen stock was centrifuged at $1620 x \mathrm{~g}$ for $15 \mathrm{~min}$, and the supernatant was collected in $1 \mathrm{ml}$ aliquots and stored at $-20^{\circ} \mathrm{C}$. The diluent used to vary antigen levels was the carbonate/bicarbonate-coating buffer ( $\mathrm{pH}$ 9.6), and the microtiter plates were Immunolon 1 type (Dynatech Laboratories, Virginia, USA). The coating of $50 \mu \mathrm{l} /$ well of the antigen was performed at $37^{\circ} \mathrm{C}$, overnight. The plate was washed four times with distilled water. The positive and negative control yolk samples were diluted to different concentrations, using different diluents, and added in 100 $\mathrm{\mu l} /$ wells. Control yolk samples were incubated at room temperature for 15 minutes. The diluted control yolk samples were discarded, and the wells were washed four times with distilled water. The conjugate used was goat antichicken IgG (light + heavy chains), labeled with a peroxidase enzyme (Kirkegaard \& Perry Laboratories, Maryland, USA). The lyophilized conjugate was reconstituted in sterile distilled water/glycerol (1:1 ratio), diluted into various concentrations using different diluents, and added at a volume of $50 \mu$ l per well. The diluted conjugate was incubated at room temperature for a period of 15 minutes, followed by four washings with distilled water. The substrate was added at 50ul/ well, and incubated at room temperature for 15 minutes. The substrate was ABTS 1-component containing 2,2'-azino-di (3 ethyl-benzthiazoline-6sulfonate) at a concentration of $0.3 \mathrm{~g} / \mathrm{L}$ in a glycine/ citric acid buffer( $\mathrm{pH} 4.0)$, supplemented with hydrogen peroxide $\left(\mathrm{H}_{2} \mathrm{O}_{2}\right)$ at a concentration of $0.02 \%$. Absorbance was read at a wavelength of $450 \mathrm{~nm}$ using Organon Teknika reader, model 530 (Organon, West Chester, PA, USA).

\section{Control yolk samples}

The negative control yolk samples were collected from eggs of 10 chicken layers kept in isolation, and that were free of Salmonella Enteritidis infection, as confirmed by daily culture of the vitelline membrane of the eggs produced from these layers during a one month period, from 27 weeks to 31 weeks of age. An equal aliquot of 50ul of yolk was pooled from 9 eggs 
collected during the last day of the 1 month-culture period, and vortexed for one minute with $450 \mu \mathrm{l}$ of sterile saline. The vortexed sample was centrifuged at $3645 x \mathrm{f}$ for 30 minutes, and the supernatant was collected and stored in small aliquots at $-20^{\circ} \mathrm{C}$.

The positive control yolk sample was collected from eggs of 10 chicken layers belonging to the same breed and flock, and kept in isolation. Each of the 10 chicken layers was challenged with $5.4 \times 10^{10} \mathrm{CFU}$ of Salmonella Enteritidis (SE) at 28 weeks of age, and the vitelline membranes of the eggs produced by the challenged layers were cultured daily for a period of 2 weekspost challenge, confirming transmission of SE in only one egg at 2 weeks post-challenge. Equal aliquots of the yolk of 8 eggs collected on the $11^{\text {th }}$ day postchallenge were pooled, and vortexed for one minute with an equal amount of sterile saline. The vortexed sample was centrifuged at $3645 \mathrm{x} g$ for 30 minutes, and the supernatant was collected and stored in small aliquots at $-20^{\circ} \mathrm{C}$.

\section{Checkerboard for development of ELISA}

The purpose of the ELISA checkerboard was to vary the different parameters included in the preliminary ELISA procedure in order to maximize the difference in the Optical Density (OD) values between positive and negative control yolk samples, targeting a difference greater than 0.350. (Veling et al., 2001; Vandekerchove et al., 2002).

Four different experiments were included in the ELISA checkerboard:

1 - The objective of experiment 1 was to study the impact of $5 \%$ Bovine Serum Albumin (BSA) dissolved in distilled water used as a blocking reagent in the step following the washing of the coated SE antigens, while applying different concentrations of positive and negative control yolk samples. In this experiment, the coated SE antigen level was fixed at $28 \mu \mathrm{g} /$ well, the conjugate was fixed at a dilution of 1:500 in Phosphate Buffered Saline (PBS), and the control yolk samples were diluted to 1.0, 1.5, 3.0, and $5.0 \%$ using PBS as a diluent. Each concentration of control yolk samples (positive or negative) was applied in quadruplicate (4 wells). The application of the diluted control yolk samples was either preceded by the blocking step or deprived from it. The volume of the blocking reagent added per well was $100 \mu \mathrm{l}$, which was incubated at room temperature for a period of 15 minutes, followed by 4 washings with distilled water.
Optical Densities (O.D.) at a wavelength of 450 $\mathrm{nm}$ of positive and negative control yolk sample in quadruplicate were recorded for each variable parameter.

2 - The objective of experiment 2 was to study the impact of $5 \%$ BSA dissolved in distilled water in the blocking step, following coating with the antigen, and a higher conjugate concentration in a new diluent of PBS-Tween 20, while applying different concentrations of positive and negative control yolk samples diluted in PBS-Tween 20. In this experiment, the coated SE antigen level was fixed at $28 \mu \mathrm{g} / \mathrm{well}$, conjugate dilution was raised to $1: 1500$ using PBS supplemented with $0.05 \%$ of Tween 20. In addition, control yolk samples were diluted to $1.0,1.5,3.0$, and $5.0 \%$, using PBS-Tween 20. Each concentration of the control yolk samples was applied in quadruplicate (4 wells). The application of the diluted control yolk samples was either preceded by the blocking step or deprived from it. Optical Densities of positive and negative control yolk samples in quadruplicate were recorded for each variable parameter.

3 - The objective of experiment 3 was to study the impact of $5 \%$ BSA dissolved in distilled water for blocking, and of diluting the conjugate at 1:1500 in either 5\% BSA dissolved in PBS-Tween 20 or PBS-Tween 20 diluent deprived of BSA, while applying different concentrations of positive and negative control yolk samples diluted in PBS-Tween 20. In this experiment, coated SE antigen level was fixed at $28 \mu \mathrm{g} / \mathrm{well}$. The control yolk samples were diluted to 1.0. 1.5, 3.0, and $5.0 \%$ using PBS-Tween 20. Each concentration of the control yolk samples was applied in duplicates ( 2 wells). The application of the diluted control yolk samples was either preceded by the blocking step or deprived from it. Optical Densities (OD) of positive and negative control yolk in duplicate were recorded for each variable parameter.

4 - The objective of experiment 4 was to study the impact of different concentrations of coated SE antigens, while blocking with 5\% BSA dissolved in distilled water, and fixing conjugate dilution at $1: 1000$ using the 5\% BSA - PBS-Tween 20 diluent, and fixing control yolk dilution at $1 \%$ in PBS-Tween 20. In this experiment, the different levels of coated SE antigen were 28, 56, 84, and $112 \mu \mathrm{g}$ protein/well. The fixed concentration of 
control yolk samples was applied in four sets, with 6 wells/set, where the respective levels of SE antigens in each set were 28, 56, 84, and $112 \mu \mathrm{g}$ protein/well. Optical Densities of positive and negative control yolk in six wells were recorded for each different SE antigen level. Results of experiments 1-4 are presented in Tables $1-4$, respectively.

\section{Preliminary application of the developed ELISA}

Based on the four experiments above, the final protocol of the developed ELISA was applied on individual egg yolk samples from each of the two groups of chicken layers: one challenged with SE in the esophagus on day 11, and the second group that wass kept in isolation, free from Salmonella infection. The respective mean O.D. values of egg yolks obtained from both groups, was statistically analyzed by OneWay ANOVA in order to evaluate the significance $(p<0.05)$ of differences in mean O.D. values of yolks obtained from the infected and uninfected layers.

\section{RESULTS AND DISCUSSION}

The absence of Tween 20 from the PBS diluent used to dilute the control yolk samples and the conjugate,

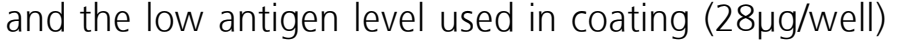
may have been responsible for the failure in differentiating Optical Density (OD) obtained from the positive or negative control yolk samples (Table 1). A higher difference in the mean absorbance O.D. values of the positive minus negative control yolk samples was obtained when the yolk was diluted at $3.0 \%$ in PBS, and the wells were blocked with $5 \%$ BSA in distilled water. However, this difference (0.134) was still lower than the targeted cutoff value of $>0.350$. It is worth noting that previous tested commercial kits presented a cutoff difference between positive and negative samples equivalent to an average O.D. value lower than 0.350 (Nicholas and Cullen, 1991; Furrer et al., 1993). The inclusion of Tween 20 at $0.05 \%$ in the diluent of the control yolk samples and in the conjugate, and lowering conjugate concentration by increasing its dilution from 1:500 to $1: 1500$ in the presence of blocking by 5\% BSA after coating, resulted in higher difference between the positive and the negative control yolk samples (Table 2 ). More specifically, the $5.0 \%$ dilution of the control yolk samples resulted in a wider difference between the positive and the negative controls (0.219), which was still lower than the targeted difference in O.D. value of $>0.350$. The detergent nature of Tween 20 may have allowed the release of the SE-antibody

\begin{tabular}{|c|c|c|c|c|}
\hline \multirow[t]{2}{*}{ Blocking treatment } & \multirow{2}{*}{$\begin{array}{l}\text { Dilutions of } \\
\text { control yolk }(\%)\end{array}$} & \multicolumn{2}{|c|}{ Mean' O.D. values of control yolk } & \multirow{2}{*}{$\begin{array}{c}\text { Difference between positive } \\
\text { \& negative O.D. values }\end{array}$} \\
\hline & & Positive & Negative & \\
\hline \multirow[t]{4}{*}{$5 \%$ BSA } & 1.0 & 2.764 & 2.735 & 0.029 \\
\hline & 1.5 & 2.740 & 2.717 & 0.023 \\
\hline & 3.0 & 2.897 & 2.763 & 0.134 \\
\hline & 5.0 & 2.750 & 2.933 & -0.183 \\
\hline \multirow[t]{4}{*}{ No BSA } & 1.0 & 2.825 & 2.753 & 0.072 \\
\hline & 1.5 & 2.760 & 2.812 & -0.052 \\
\hline & 3.0 & 2.894 & 2.819 & 0.075 \\
\hline & 5.0 & 2.825 & 2.946 & -0.121 \\
\hline
\end{tabular}

a - Coating per well is equivalent to $28 \mu \mathrm{g}$ of SE protein. $\mathrm{b}$ - Yolk was diluted at 1.0-5.0 \% in PBS, and the conjugate was diluted at $1: 500$ in PBS.

c - Mean of quadruplicates.

\begin{tabular}{|c|c|c|c|c|}
\hline \multirow[t]{2}{*}{ Blocking treatment } & \multirow{2}{*}{$\begin{array}{l}\text { Dilutions of } \\
\text { control yolk }(\%)\end{array}$} & \multicolumn{2}{|c|}{ Meanc O.D. values of control yolk } & \multirow{2}{*}{$\begin{array}{c}\text { Difference between positive } \\
\& \text { negative O.D. values }\end{array}$} \\
\hline & & Positive & Negative & \\
\hline \multirow[t]{4}{*}{$5 \%$ BSA } & 1.0 & 0.981 & 0.881 & 0.100 \\
\hline & 1.5 & 1.104 & 0.903 & 0.201 \\
\hline & 3.0 & 1.140 & 0.931 & 0.209 \\
\hline & 5.0 & 1.156 & 0.937 & 0.219 \\
\hline \multirow[t]{4}{*}{ No BSA } & 1.0 & 0.975 & 0.886 & 0.089 \\
\hline & 1.5 & 1.029 & 0.891 & 0.138 \\
\hline & 3.0 & 1.037 & 0.884 & 0.153 \\
\hline & 5.0 & 1.127 & 0.929 & 0.198 \\
\hline
\end{tabular}

a - Coating per well is equivalent to $28 \mu \mathrm{g}$ of SE protein. b - Yolk was diluted at 1.0-5.0 \% in PBS, and the conjugate was diluted at $1: 500$ in PBS. c - Mean of quadruplicates 
component from the remaining yolk components, or else, it could have created an optimal ionic environment the binding of yolk antibodies and conjugate antibodies to the coated SE antigens, and to the captured chicken antibodies, respectively (Lee et al., 2003; Halim et al., 2005).

Results of the comparison of the effect of the nature of the diluent used for the conjugate (5\% BSA in PBSTween 20 versus PBS-Tween alone) in the presence or absence of blocking by $5 \%$ BSA, while using control yolk samples in dilutions between $1.0-5.0 \%$ in PBSTween 20, are shown in Table 3. The maximum difference in the O.D. values between the positive and the negative yolk samples (0.279) was obtained when the yolk was diluted to $1.0 \%$ in PBS-Tween 20, and the conjugate was diluted in $5 \%$ BSA in PBS-Tween 20. It was shown that this improvement in O.D. value differences between positive and negative was due to the inclusion of $5 \%$ BSA in the PBS-Tween 20 diluent used for the conjugate. BSA at 5\% may have blocked non-specific sites in the microtiter plate well, thereby increasing the specificity of the SE-antibodies present in the positive control yolk samples, or preventing antibodies in the negative control yolk sample that are not specific to SE to bind to the coated antigens (Vandekerchove et al., 2002).

The objective of the assay presented in Table 4 was to obtain a difference between positive and negative control yolk O.D. values higher than 0.350. The variation in this assay was the change in the SE protein level used for coating, which ranged from 28 to $112 \mu \mathrm{g} /$ well, while yolk dilution was kept constant at $1.0 \%$ in PBS-Tween 20, as well as conjugate dilution at 1:1000 in 5\% BSA dissolved in PBS-Tween 20. Results in Table 4 show that the maximal difference in O.D. values was 0.381 between the positive and the negative control yolk samples, at an antigen level of $112 \mu \mathrm{g} /$ well. This difference was higher than the targeted cutoff of 0.350 . The ELISAs developed by other researcher presented O.D. value differences between positive and negative control samples equal or higher than 0.300 (Barrow, 1994; Desmidt et al., 1996; DeraTomaszewska et al., 2003; Oliveira et al., 2004).

Interestingly, in spite of achieving the objective of obtaining significant differences in OD values between positive and negative control yolk samples, the negative control yolk samples still presented a high background optical density value of 0.862 (Table 4). Future investigations should target a lower OD value for negative control yolk samples, which could be achieved by increasing yolk sample dilution over $1 \%$ (VanderSchalie et al., 1994; Clavijo et al., 1995).

In conclusion, the final protocol of the ELISA for the quantification of SE-specific antibodies in the egg yolk of chickens based on the above data, presented in Tables 1-4, is to coat the microtiter plate wells with $112 \mu \mathrm{g} /$ well of SE proteins, to block them using 5\% BSA in distilled water, and to dilute the yolk samples at $1 \%$ in PBS-Tween 20, and the conjugate at 1:1000 in 5\%BSA dissolved in PBS-Tween 20.

The mean O.D. values of SE-specific antibodies in individual egg yolks, obtained by SE-challenged and

\begin{tabular}{|c|c|c|c|c|c|c|}
\hline \multirow{2}{*}{$\begin{array}{l}\text { Treatment } \\
\text { Blocking } \frac{}{5}\end{array}$} & \multicolumn{2}{|c|}{ Nature of conjugate diluent } & \multirow{2}{*}{$\begin{array}{c}\text { Dilution of } \\
\text { of control yolk (\%) }\end{array}$} & \multicolumn{2}{|c|}{$\begin{array}{l}\text { Meand O.D. values } \\
\text { control yolk }\end{array}$} & \multirow{2}{*}{$\begin{array}{l}\text { Difference between positive } \& \\
\text { negative O.D. values }\end{array}$} \\
\hline & BSA in PBS-Tween & 20 PBS-Tween 20 & & Positive & Negative & \\
\hline \multirow[t]{8}{*}{$5 \%$ BSA } & + & & 1.0 & 1.234 & 0.955 & 0.279 \\
\hline & + & & 1.5 & 1.208 & 1.040 & 0.168 \\
\hline & + & & 3.0 & 1.280 & 1.070 & 0.210 \\
\hline & + & & 5.0 & 1.202 & 1.134 & 0.068 \\
\hline & & + & 1.0 & 1.270 & 1.091 & 0.179 \\
\hline & & + & 1.5 & 1.157 & 1.103 & 0.054 \\
\hline & & + & 3.0 & 1.314 & 1.053 & 0.261 \\
\hline & & + & 5.0 & 1.288 & 1.106 & 0.182 \\
\hline \multirow[t]{8}{*}{ No BSA } & + & & 1.0 & 1.143 & 0.978 & 0.166 \\
\hline & + & & 1.5 & 1.239 & 1.039 & 0.200 \\
\hline & + & & 3.0 & 1.280 & 1.112 & 0.168 \\
\hline & + & & 5.0 & 1.194 & 1.115 & 0.079 \\
\hline & & + & 1.0 & 1.145 & 0.983 & 0.163 \\
\hline & & + & 1.5 & 1.185 & 0.991 & 0.194 \\
\hline & & + & 3.0 & 1.175 & 1.007 & 0.168 \\
\hline & & + & 5.0 & 1.124 & 0.992 & 0.132 \\
\hline
\end{tabular}

a - Coating per well is equivalent to $28 \mu \mathrm{g}$ of SE protein. $\mathrm{b}$ - Conjugate dilution at 1:1500. c - Control yolk samples were diluted in PBS-Tween 20 at 1.0-5.0\%. d-Mean of duplicates. 
control-unchallenged layers, were 0.8578 and 0.5250 $(p<0.05)$ (Table 5$)$. Future investigations will evaluate the sensitivity of this developed ELISA for the detection of SE-specific antibodies in chicken yolk at different intervals after a controlled challenge, and for the study the dynamics of the infection titers through the production cycle of infected flocks. In addition, future investigations could implement this developed ELISA to evaluate different eradication or control programs of SE infections in poultry as a prerequisite for their control in food-borne human diseases.

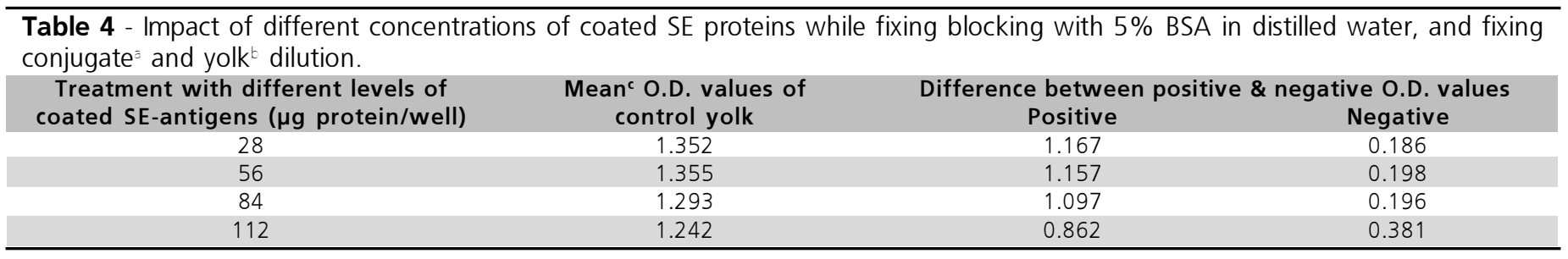

a - Conjugate was diluted at 1:1000 in 5\% BSA dissolved in PBS-Tween 20. b - Yolk dilution was fixed at $1 \%$ in PBS-Tween 20 . c - Mean of six replicates.

Table 5 - Individual O.D. values of SE-specific antibodies in egg yolks obtained from SE-challenged ${ }^{1}$ and control-unchallenged layers.

\begin{tabular}{ccc} 
& \multicolumn{2}{c}{ O.D. values from layers } \\
\cline { 2 - 3 } Number of eggs & SE-challenged & Control-unchallenged \\
\hline 1 & 0.784 & 0.523 \\
2 & 0.769 & 0.566 \\
3 & 0.921 & 0.555 \\
4 & 0.848 & 0.390 \\
5 & 0.712 & 0.426 \\
6 & 0.846 & 0.550 \\
7 & 0.840 & 0.623 \\
8 & 0.911 & 0.567 \\
9 & 1.089 & - \\
Mean & $0.8578^{3}$ & $0.5250^{b}$ \\
\hline
\end{tabular}

1 - Eggs collected on the 11 th day post-challenge from layers challenged in the esophagus with SE at $5.4 \times 1010 \mathrm{CFU} / \mathrm{ml} / \mathrm{bird}$. a-b Means in the same row followed by different superscripts are significantly different $(p<0.05)$.

\section{REFERENCES}

Barbour EK, Hamadeh SK, Zoubiane G, Talhouk R, Hilan C. An Enzyme-Linked Immunosorbent Assay for evaluation of an experimental Salmonella typhimurium vaccine in two breeds of ewes. Small Ruminant Research 1996; 21:239-244.

Barbour EK, Jurdi LH, Talhouk R, Qatani M, Eid A, SakrW, Bouljihad M, Spasojevic R. Emergence of Salmonella Enteritidis outbreaks in broiler chickens in the Lebanon: epidemiological markers and competitive exclusion control. Revue Scientifique et Technique (Office International des Epizooties) 1999; 18(3):710-718.

Barrow PA. Serological diagnosis of Salmonella serotype enteritidis infections in poultry by ELISA and other tests. International Journal of Food Microbiology 1994; 21:55-68

Clavijo A, Thorsen J. Bacterial expression of the caprine arthritisencephalitis virus gag and env proteins and their use in enzymelinked immunosorbent assay. American Journal of Veterinary Research 1995; 56:841-848.
Dadrast H, Hesketh R, Taylor DJ. Egg yolk antibody detection in identification of Salmonella infected poultry. The Veterinary Record 1990; $126: 219$

Dera-Tomaszewska B, Wysocki J, Kunikowska D, Dziadziuszko H, Giosnicka R. Hsp60 specific antibodies in egg yolks from laying hens naturally infected with Salmonella enterica subspecies enterica serovar Enteritidis. Comparative Imunology, Microbiology and Infectious Diseases 2003; 26 (1):37-45.

Desmidt M, Ducatelle R, Haesebrouck F, de Groot PA, Verlinden M, Wijffels R, Hinton M, Bale JA, Allen VM. Detection of antibodies to Salmonella Enteritidis in sera and yolk from experimentally and naturally infected chickens. Veterinary Record 1996; 138:223-226.

Furrer B, Baumgartner A, Bommeli W. Enzyme-Linked Immunosorbent Assay (ELISA) for the detection of antibodies against Salmonella Enteritidis in chicken blood or egg yolk. Zentralblatt fur Bakteriologie 1993; 279:191-200.

Gast RK, Beard CW. Age-related changes in the persistence and pathogenicity of Salmonella typhimurium in chicks. Poultry Science 1989; 68:1454-1460.

Gast RK, Porter RE, Holt PS. Assessing the sensitivity of egg yolk antibody testing for detecting Salmonella Enteritidis infections in laying hens. Poultry Science 1989; 76:798-801.

Gorham SL, Kadavil K, Vaughan E, Lambert H, Abel J, Pert B. Gross and microscopic lesions in young chickens experimentally infected with Salmonella Enteritidis. Avian Diseases 1994: 38(4):816-21.

Gruenewald R, Carter RJ, Singh TP, Terry S, Williams G, Ramon A. Prevalence of Salmonella enterica serovar Enteritidis and other Salmonella serovars among immunocompromised and immunocompetent persons in New York City. In: Saeed A.M., Gast R.K., Potter M.E., Wall P.G., editors. Salmonella enterica serovar Enteritidis in humans and animals. Ames: lowa State University Press; 1993-95. p.117-122

Guard-Petter J. Variants of smooth Salmonella enterica serovar Enteritidis that grow to higher cell density than the wild type are more virulent Applied and Environmental Microbiology 1998; 64(6): 2166-2172. 


\section{Tayeb IT, Nehme P, Jaber L,} BarbourEK
Development of an Enzyme-Linked Immunosorbent Assay for quantification of Salmonella Enteritidis-specific antibodies in egg yolk
Guard-Petter J, Parker CT, Asokan K, Carlson RW. Clinical and veterinary isolates of Salmonella enterica serovar Enteritidis defective in lipopolysaccharide O-chain polymerization. Applied and Environmental Microbiology 1999; 65(5):2195-2201.

Halim ND, Joseph AW, Lipska BK A novel ELISA using PVDF microplates. Journal of Neuroscience Methods 1005; 143(2):163168.

Hoop RK, Pospischil A. Bacteriological, serological, histological and immunohistochemical findings in laying hens with naturally acquired Salmonella Enteritidis phage type 4 infection. Veterinary Record 1993; 133(16):391-393.

Lee W, Oh B-K, Min Bae Y, Paek S., Lee WH, Choi J-W. Fabrication of self-assembled protein $A$ monolayer and its application as an immunosensor. Biosensors and Bioelectronics 2003; 19(3):185192.

Nicholas RA, Cullen GA. Development and application of an ELISA for detecting antibodies to Salmonella enteritidis in chicken flocks. Veterinary Record 2003; 128:74-76.

Okamura M, Kamijima $Y$, Miyamoto $T$, Tani H, Sasai K, Baba E. Differences among six salmonella serovars in abilities to colonize reproductive organs and to contaminate eggs in laying hens. Avian Diseases 2001; 45: 61-69.

Oliveira GH de, Berchieri Junior A, Montassier HJ, Fernades AC Assessment of serological response of chickens to Salmonella gallinarum and Salmonella pullorum by ELISA. Revista Brasileira de Ciência Avícola 2004; 6 (2):111-115.

Saeed AM, Nair US. Use of molecular biological markers in the epidemiological study of Salmonella enterica serovar Enteritidis infections in humans and animals. In: Saeed AM, Gast RK, Potter ME, Wall PG, editors. Salmonella enterica serovar Enteritidis in humans and animals. Ames: lowa State University Press; 1999. p.161-170.

Uyttebroek LA, Devriese A, Derore A, Ducatelle R, Haesebrouck F. Fecal shedding of Salmonella enteritidis in experimentally infected replacement pullets: persistence of colonization and effects of previous infection. In: Ducatelle R., Haesebrouck F., Mulder R.W.A.W., editors. Proceedings FLAIR/COST NO 906: Salmonella Colonization in Poultry Gent; 1991. p.21-31.

Van Asten A, Van Dijk JE. Distribution of "classic" virulence factors among Salmonella spp. FEMS Immunology and Medical Microbiology 2005; 44(3):251-259.

Vandekerchove DGF, Kerr PG, Callebaut AP, Ball HJ, Stakenborg T, Marien J, Peeters JE. Development of a capture ELISA for the detection of antibodies to enteropathogenic Escherichia coli(EPEC) in rabbit flocks using intimin-specific monoclonal antibodies. Veterinary Microbiology 2005; 88(4):351-366.

VanderSchalie J., Bradway D.S., Besser T.E., Evermann J.F. Evaluation of a kinetic enzyme-linked immunosobent assay for detection of caprine arthritis-encephalitis virus specific antibodies. Journal of Veterinary Diagnostic Investigation 1994; 6:30-33.
Veling J, Van Zijderveld FG, Van Zijderveld-van Bemmel AM, Schukken YH, Barkema HW. Evaluation of two enzyme-Linked Immusorbent Assays for detecting Salmonella enterica subsp. Enterica Serovar Dublin antibodies in bulk milk. Clinical and Diagnosis Laboratory Immunology 2001; 8(6):1049-1055. 